BMJ Open

Diabetes

Research

\& Care

\title{
Trends in inpatient admissions and emergency department visits for heart failure in adults with versus without diabetes in the USA, 2006-2017
}

\author{
Jessica L Harding (D) , , ${ }^{1,2}$ Stephen R Benoit (D) , ${ }^{3}$ Israel Hora, ${ }^{3}$ \\ Lakshmi Sridharan (1) , ${ }^{4}$ Mohammed K Ali, ${ }^{3,5,6}$ Ram Jagannathan, ${ }^{2}$ \\ Rachel E Patzer, ${ }^{1} \mathrm{~K}$ M Venkat Narayan (D) ${ }^{5}$
}

To cite: Harding JL, Benoit SR, Hora I, et al. Trends in inpatient admissions and emergency department visits for heart failure in adults with versus without diabetes in the USA, 2006-2017. BMJ Open Diab Res Care 2021;9:e002377. doi:10.1136/ bmjdrc-2021-002377

- Additional supplemental material is published online only. To view, please visit the journal online (http://dx.doi. org/10.1136/bmjdrc-2021002377)

Received 11 May 2021 Accepted 22 September 2021

Check for updates

(C) Author(s) (or their employer(s)) 2021. Re-use permitted under CC BY-NC. No commercial re-use. See rights and permissions. Published by BMJ.

For numbered affiliations see end of article.

Correspondence to Dr Jessica L Harding; jessica.harding@emory.edu

\section{ABSTRACT}

Introduction Heart failure (HF) is a major contributor to cardiovascular morbidity and mortality in people with diabetes. In this study, we estimated trends in the incidence of HF inpatient admissions and emergency department (ED) visits by diabetes status.

Research design and methods Population-based agestandardized HF rates in adults with and without diabetes were estimated from the 2006-2017 National Inpatient Sample, Nationwide ED Sample and year-matched National Health Interview Survey, and stratified by age and sex. Trends were assessed using Joinpoint.

Results HF inpatient admissions did not change in adults with diabetes between 2006 and 2013 (from 53.9 to 50.4 per 1000 persons; annual percent change (APC): -0.3 (95\% Cl -2.5 to 1.9) but increased from 50.4 to 62.3 between 2013 and 2017 (APC: 4.8 (95\% Cl 0.3 to 9.6)). In adults without diabetes, inpatient admissions initially declined (from 14.8 in 2006 to 12.9 in 2014; APC -2.3 $(95 \% \mathrm{Cl}-3.2$ to -1.2$))$ and then plateaued. Patterns were similar in men and women, but relative increases were greatest in young adults with diabetes. HF-related ED visits increased overall, in men and women, and in all age groups, but increases were greater in adults with (vs without) diabetes.

Conclusions Causes of increased HF rates in hospital settings are unknown, and more detailed data are needed to investigate the aetiology and determine prevention strategies, particularly among adults with diabetes and especially young adults with diabetes.

\section{INTRODUCTION}

People with diabetes are at increased risk for cardiovascular disease (CVD) and associated complications. ${ }^{1}$ Although diabetes has become an increasingly common disease, estimated to affect 463 million people worldwide $^{2}$ and more than 34 million in the USA, ${ }^{3}$ CVD and related mortality in people with diabetes has fallen dramatically in most high-income countries since the 1980s likely due to advances in treatment and better management of risk factors. ${ }^{45}$ However, the

SIGNIFICANCE OF THIS STUDY
WHAT IS ALREADY KNOWN ABOUT THIS
SUBJECT?
$\Rightarrow$ Heart failure (HF) is a major contributor to cardio-
vascular morbidity and mortality in people with
diabetes.
$\Rightarrow$ Whether HF-related hospitalizations among adults
with versus without diabetes has changed over time
remains unknown.
WHAT ARE THE NEW FINDINGS?
$\Rightarrow$ Rates of HF-related inpatient admissions and ED
visits are three to five times higher in adults with
versus without diabetes, and this excess risk has
increased over time.
$\Rightarrow$ Though absolute rates remain lowest in the young-
est age groups, the greatest relative increases in
HF-related inpatient admissions and ED visits were
observed in young adults with diabetes.
$\Rightarrow$ Increases in HF-related utilization among adults with
diabetes was observed in both inpatient and ED set-
tings, suggesting broader underlying causes rather
than a shift in treatment setting.
HOW MIGHT THESE RESULTS CHANGE THE
FOCUS OF RESEARCH OR CLINICAL PRACTICE?
$\Rightarrow$ Combined with current evidence from clinical trials,
findings of this study support the use of intensive
and focused prevention and management of diabe-
tes, including the use of SGL2 inhibitors, to reduce
the incidence of HF hospitalizations in people with
diabetes.
$\Rightarrow$ Future research should focus on the drivers of in-
creases in HF hospitalizations, especially among
young people with diabetes.

reported declines in CVD among people with diabetes (both incidence and mortality) often do not include heart failure (HF) as an outcome, despite increasing recognition that $\mathrm{HF}$ is a major contributor to CVD 
morbidity, mortality and healthcare costs in people with diabetes. ${ }^{16-11}$

In a 2015 paper, Shah $e t a t^{\ominus}$ demonstrated that HF is more likely to be an initial manifestation of CVD in people with type 2 diabetes compared with myocardial infarction, stroke and coronary disease. Despite the relative importance of HF in diabetes, few studies have comprehensively examined whether rates of HF in people with diabetes (vs without diabetes) has changed over time. In the USA, one recent study demonstrated that HF inpatient admissions, defined as the primary reason for hospital admission, increased 3.6\% per year between 2013 and 2015 following a period of decline. ${ }^{12}$ However, to understand the underlying drivers of changes in HF rates and develop subsequent interventions, a comparison with people without diabetes is needed. Such comparisons in atherosclerotic CVD (eg, myocardial infarction and coronary artery disease) have led to narrowing the gap by reducing the excess risk in diabetes populations. ${ }^{13}{ }^{14}$ Furthermore, a more comprehensive approach to understanding the overall HF burden is necessary to inform healthcare planning and resource allocation. This includes consideration of multiple settings in which HF care is likely to occur, as well as consideration of $\mathrm{HF}$ as both a primary and contributory cause for hospitalization.

Using nationally representative USA data, we estimated secular trends in the incidence of HF-related inpatient admissions and ED visits among adults with diabetes versus adults without diabetes between 2006 and 2017 .

\section{METHODOLOGY}

The National Inpatient Sample (NIS) and the Nationwide Emergency Department Sample (NEDS)

We analyzed annual data (2006-2017) from the Agency for Healthcare Research and Quality's NIS and NEDS. ${ }^{15}$ NIS and NEDS, the largest all-payer inpatient and ED databases in the USA, includes 7 million and 30 million unweighted annual visits, respectively. ${ }^{15}$ Both data sets approximate a $20 \%$ stratified sample of discharges and can be weighted to provide nationally representative estimates. Rehabilitation and long-term acute care hospitals are excluded from NIS. Both NIS and NEDS include International Classification of Diseases Clinical Modification (ICD-CM) diagnostic codes as well as patient demographics, hospital characteristics, payment sources, patient disposition and total charges. Both NIS and NEDS data represent hospital discharges, not individual persons, and therefore our analysis does not account for multiple admissions per person.

A hospitalization was considered to be related to $\mathrm{HF}$ if at least one ICD-9-CM diagnosis code 428.x between January 2000 and September 2015, or ICD-10-CM diagnosis code I50.x between October 2015 and December 2017, appeared in NIS or NEDS data. This approach is aimed to better capture the overall burden of HF by including HF listed as the primary or contributory cause of the hospitalization. In a sensitivity analysis, we defined
HF as the primary cause of hospital admission in NIS and NEDS between January 2006 and September 2015. This analysis was restricted to September 2015 and earlier due to known coding changes implemented in October 2015 that impacted the likelihood of HF being listed as the primary cause of hospital admissions in later years. ${ }^{16}$ The 2015 population data (from National Health Interview Survey (NHIS)) were weighted by 0.75 to reflect that only three-quarters of the numerator data was used. ${ }^{17}$ To avoid double-counting, we excluded ED visits where the disposition was admission to the hospital because these HF events were accounted for in the inpatient data. Each HF-related admission was considered to be related to diabetes if any of the listed diagnoses also included a diabetes code (ICD-9-CM: 250 .x, 357.2, 366.41; ICD10-CM: E10, E11 and E13). Comorbidities, adapted from the Charlson Comorbidity Index, among hospitalized patients with $\mathrm{HF}$ and with or without diabetes were defined using ICD-9-CM and ICD-10-CM, as appropriate (see online supplemental table 1 ).

\section{The National Health Interview Survey}

Using annual data (2006-2017) from the NHIS, we estimated the number of persons aged $\geq 18$ years with and without diabetes. ${ }^{18}$ The NHIS is a household-based survey of the health of the civilian, non-institutionalized USA population. ${ }^{18}$ We defined adults with diabetes if the sample adult responded yes to the question, 'other than during pregnancy, have you ever been told by a doctor or other health professional that you have diabetes or sugar diabetes?'. This survey does not distinguish between diabetes types; but given that type 2 diabetes accounts for $90 \%-95 \%$ of all diabetes cases, ${ }^{19}$ we consider the results of this study to be generalizable to people with diagnosed type 2 diabetes. Data from the NHIS were weighted to make estimates representative of the demographic characteristics of the US civilian noninstitutionalized population.

\section{Statistical analysis}

We reported the crude weighted number of patients with HF at the start (2006), middle (2011) and end (2017) of the study period, stratified by diabetes status, and age group, sex, location (urban, micropolitan and rural), household income (quartiles), USA region (northeast, midwest, south and west) and comorbidities for both NIS and NEDS. The weighted results estimate the number of inpatient admissions and non-admission ED visits in the USA due to HF.

Annual rates were calculated as the number of HF hospitalizations with and without diabetes (as determined from NIS and NEDS), divided by the number of persons with and without diabetes (as determined from NHIS). We reported age-standardized rates of HF per 1000 adults with diabetes and per 1000 adults without diabetes. Age (grouped into $18-44,45-64,65-74$ and $\geq 75$ years) and sex-specific rates were also calculated. Rates were age standardized using the 2000 USA standard population. Excess risk between diabetes and non-diabetes populations was estimated as rate ratios 


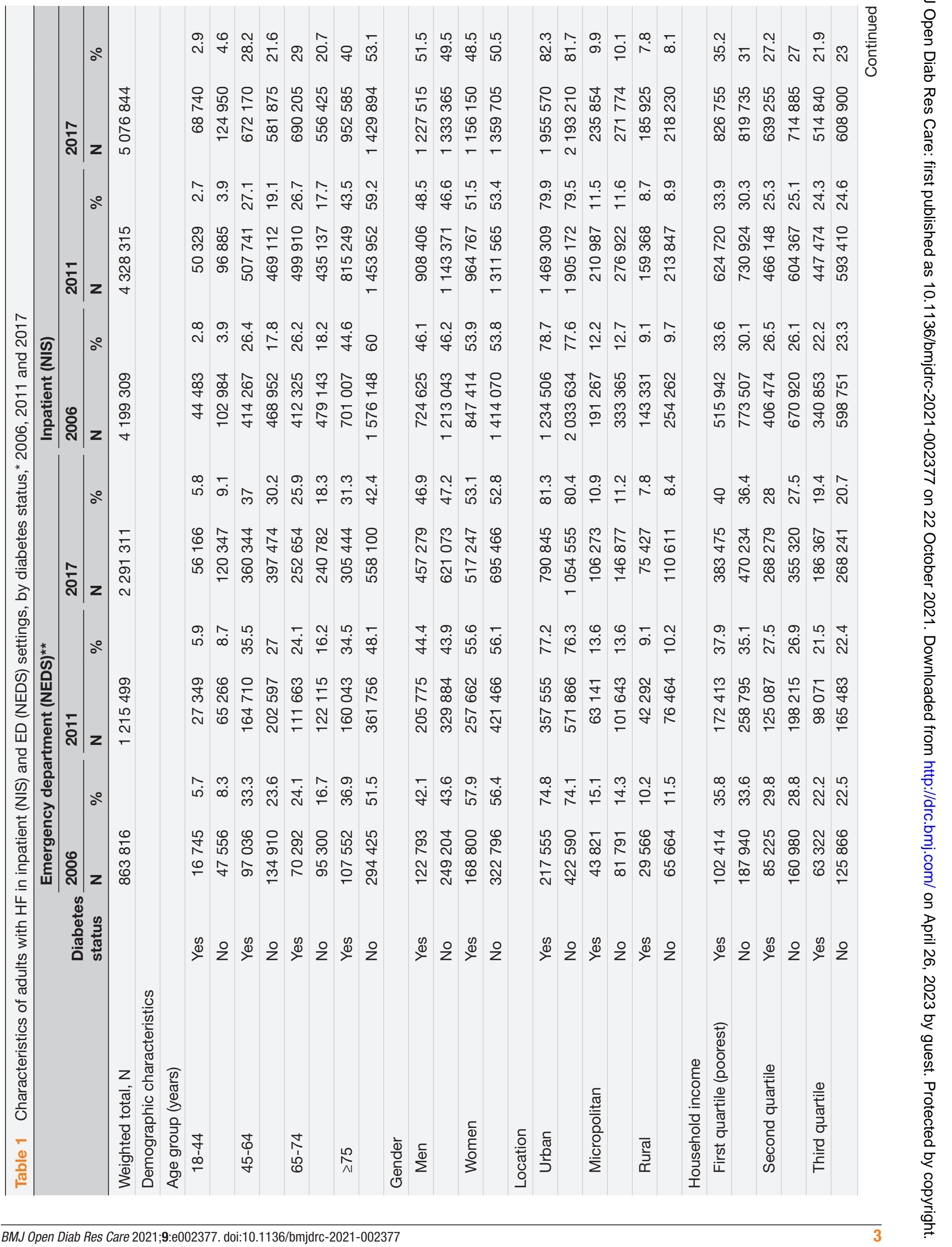




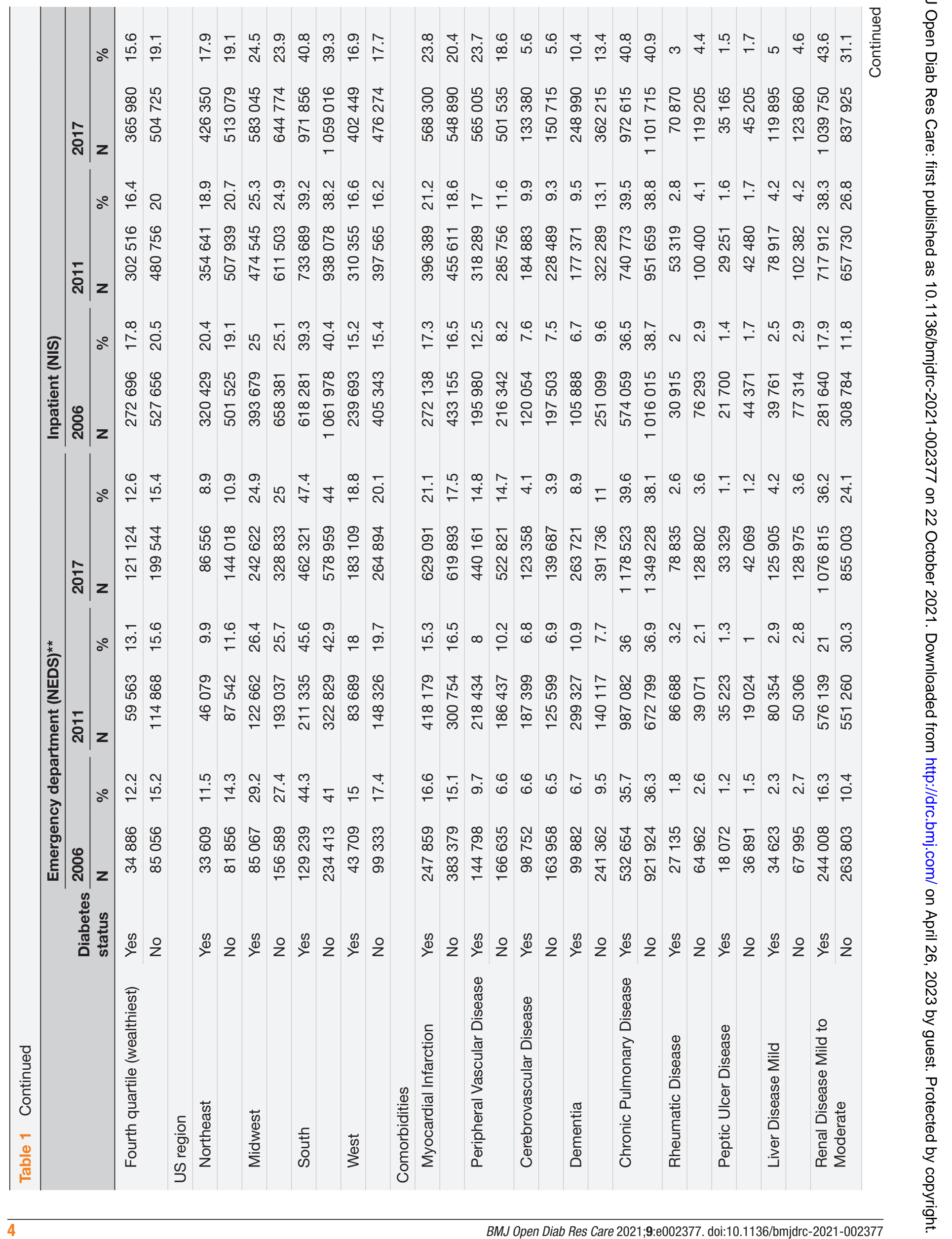




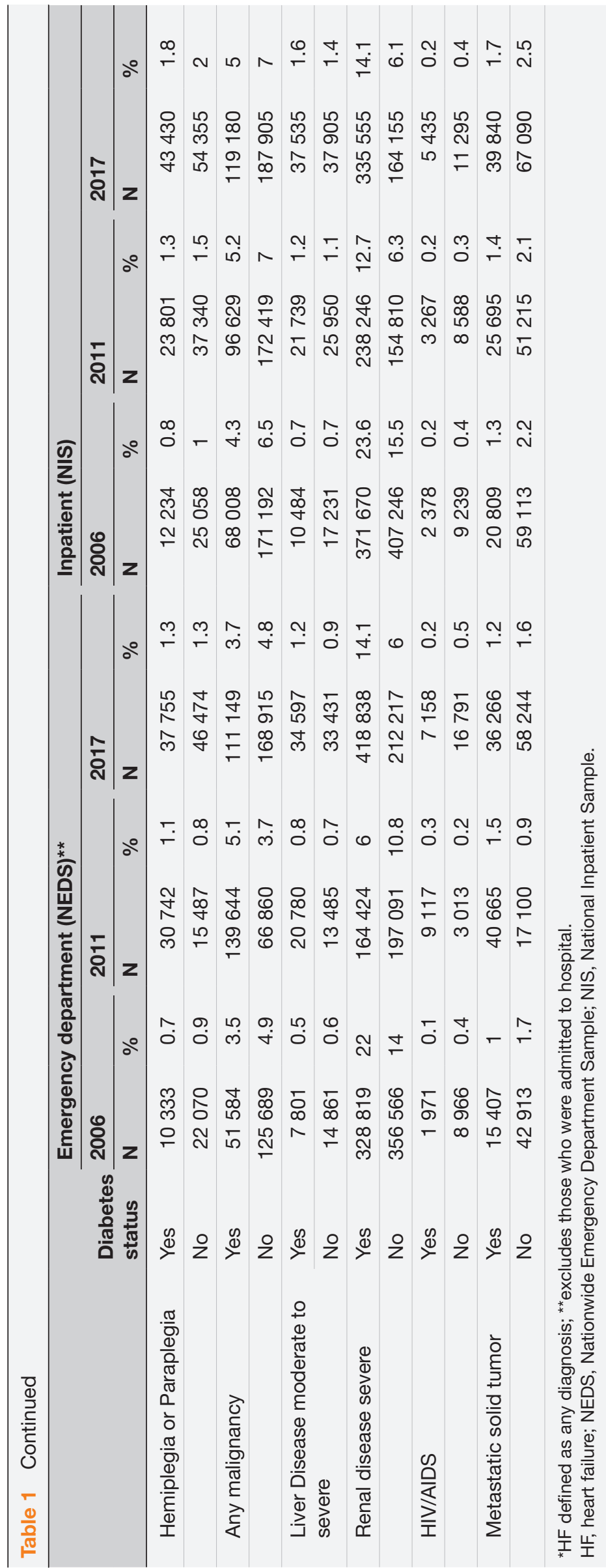


(RRs). We used SAS-callable SUDAAN (RTI International) to account for the complex sampling design in NIS, NEDS and NHIS, and the Taylor series linearization was used to estimate the variance of the ratio of the numerator and denominator. The delta method was used to compute SEs and $95 \%$ CIs for rates and RRs accounting for the weighted design of NIS, NEDS and NHIS. ${ }^{20}$

In 2012, the NIS sampling design was changed, which has implications for trend analyses. Per NIS guidelines, we used NIS-provided trend weights for the years preceding 2012 and the discharge weights beginning in 2012 to make the discharge outcome consistent with the new sampling design. ${ }^{21}$

Joinpoint regression was used to examine trends over time. ${ }^{22}$ This software uses permutation tests to identify points where linear trends change significantly in either direction or magnitude and calculates an annual percentage change (APC) for each time period identified. A maximum of two joinpoints were specified. A $p$ value of $<0.05$ was established as statistical significance.

\section{RESULTS}

Characteristics of adults with HF in inpatient (NIS) and ED (NEDS) settings in 2006, 2011 and 2017, and by diabetes status, are described in table 1 . In brief, among HF-related inpatient admissions and ED visits between 2006 and 2017, there was an increase in the proportion of men, middle-age adults (aged 45-64 and 65-74 years), adults residing in urban settings, adults reporting low-income households and adults living in the West. This was broadly true for people with and without diabetes. In addition, the proportion of $\mathrm{HF}$ hospitalizations, both in ED and inpatient settings, with comorbidities increased in people with and without diabetes with a few exceptions: HF-related hospitalizations with cerebrovascular disease and severe renal disease decreased over time in people with and without diabetes, and the proportion of HF-related hospitalizations with peptic ulcer disease, HIV, malignancy or metastatic solid tumor did not change over time in people with or without diabetes. The increasing proportion of most comorbidities was, generally, higher in people with as compared without diabetes.

\section{National Inpatient Sample}

In 2017, rates of HF-related inpatient admissions were more than five times as high in adults with versus without diabetes (RR: 5.1 (95\% CI 4.7 to 5.5)), a significant increase from 3.6 (95\% CI 3.3 to 4.0 ) in 2006 (table 2). Overall, between 2006 and 2013, rates of HF-related inpatient admissions did not change among adults with diabetes, and then increased sharply between 2013 and 2017 from 50.4 to 62.3 per 1000 persons (APC: 4.8 (95\% CI 0.3 to 9.6) (figure 1A and table 2). Among adults without diabetes, the opposite was observed: between 2006 and 2014, rates declined from 14.8 to 11.7 (APC -2.3 (95\% CI -3.2 to -1.2 ) and plateaued thereafter. Similar patterns were observed in both men and women.

By age, differences were noted (figure 2A and table 2). First, the excess risk associated with diabetes decreased with increasing age. For example, the $2017 \mathrm{RR}$ was 20.2 (95\% CI 16.9 to 23.5 ) versus 2.8 (95\% CI 2.5 to 3.2) for those aged $18-44$ and $\geq 75$ years, respectively. Second, in adults aged 18-44 years with and without diabetes, rates of HF-related inpatient admissions increased similarly such that there was no significant change in the excess risk associated with diabetes over time. Third, among adults aged 45-64 and 65-74 years with and without diabetes, HF rates increased after a period of decline and the excess risk associated with diabetes increased. Last, among adults aged $\geq 75$ years, rates of HF-related inpatient admissions declined throughout the study period in adults without, but not with, diabetes and the excess risk associated with diabetes increased (from RR of 2.0 to 2.8; APC 2.4 (95\% CI 0.6 to 4.2$)$ ).

\section{Nationwide Emergency Department Sample}

In 2017, rates of HF-related ED visits were more than five times as high in adults with versus without diabetes (RR: $5.2(95 \%$ CI 4.5 to 5.9$)$ ), a significant increase from 3.7 (95\% CI 3.2 to 4.1 ) in 2006 (table 3). Overall, between 2006 and 2017, rates of HF-related ED visits increased in adults with (from 11.5 to 43.6 per 1000 persons) and without (from 3.1 to 5.9 per 1000 persons) diabetes (figure 1B and table 3). However, the rate of increase was greater in adults with diabetes, leading to an increase in the excess risk of HF-related ED visits associated with diabetes over.

Increases in HF-related ED visits were observed across all age groups and in adults with and without diabetes (figure 2B and table 3). For all age groups, excluding 65-74 years, the excess risk associated with diabetes did not significantly change over time, indicating increasing rates of $\mathrm{HF}$ ED visits were similar in adults with and without diabetes. However, among adults aged 65-74 years, the HF rate increase was greater in adults with diabetes, leading to an increase in the excess risk associated with diabetes over time (from RR of 3.3 to 4.4; APC $2.0(95 \%$ CI 0.7 to 3.3$))$.

\section{Sensitivity analyses}

In a sensitivity analysis, we examined trends in HF inpatient admissions and ED visits between 2006 and 2015 where HF was defined as the primary reason for the admission (online supplemental tables 2 and 3). Overall, in 2015 rates of primary inpatient $\mathrm{HF}$ admissions and HF ED visits were 4.7 (95\% CI 4.4 to 5.1 ) and $3.2(95 \%$ CI 2.8 to 3.5) times as high in adults with versus without diabetes, respectively.

Though absolute rates were substantially lower when HF was defined as the primary (vs any) reason for admission, inpatient patterns were similar insofar as the excess risk associated with diabetes, particularly among younger adults, increased over time (online supplemental table 2). 


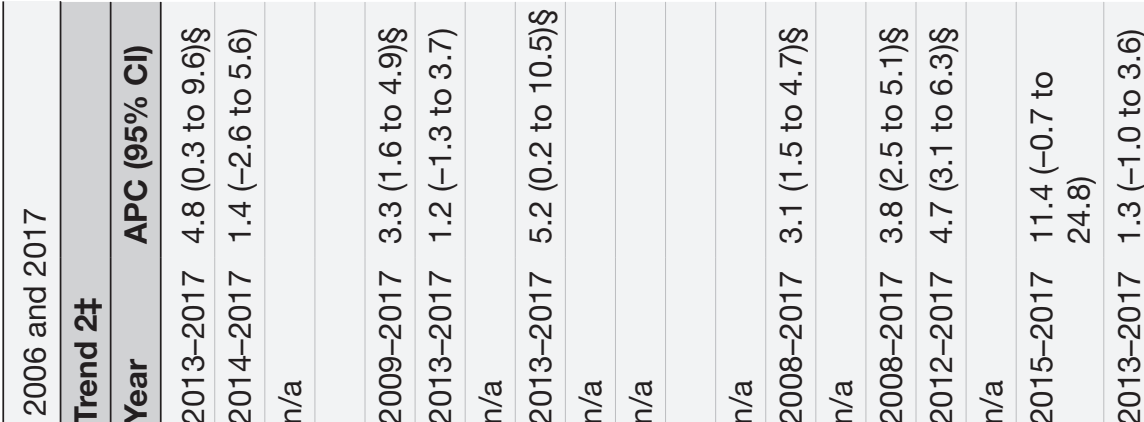

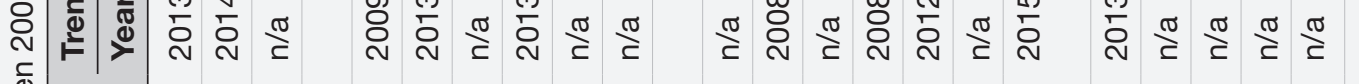

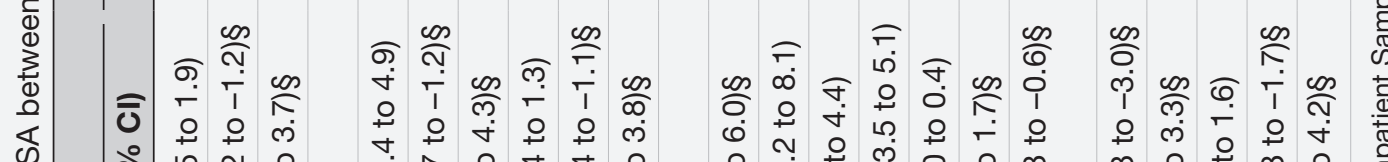

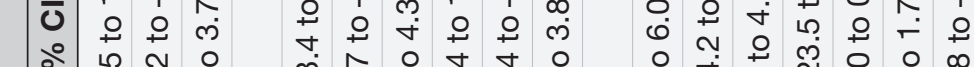

ᄀ 낭

。 I I

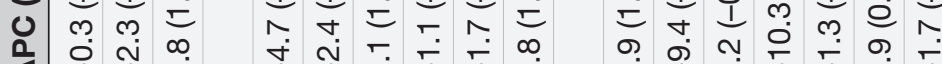

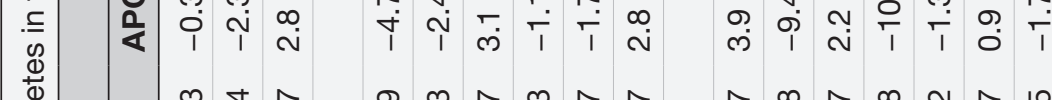

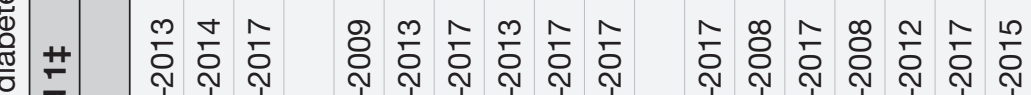

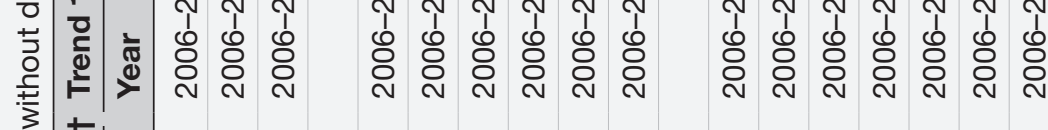

○

m.

is

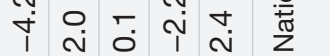

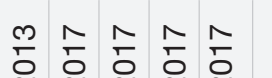

สิ จे จे จे

\& 8 \&े

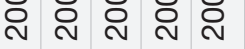

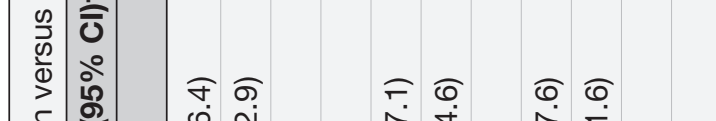

ลุ

क $\quad$ ब

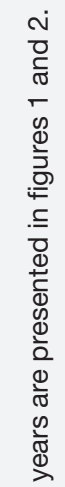

ปิ

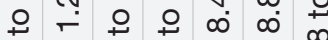

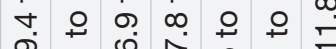

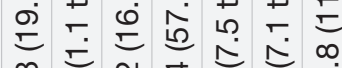

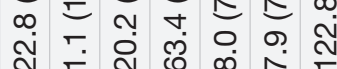

至

N

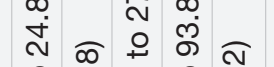

ำ

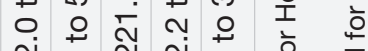

ลู่

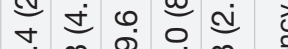

N

o

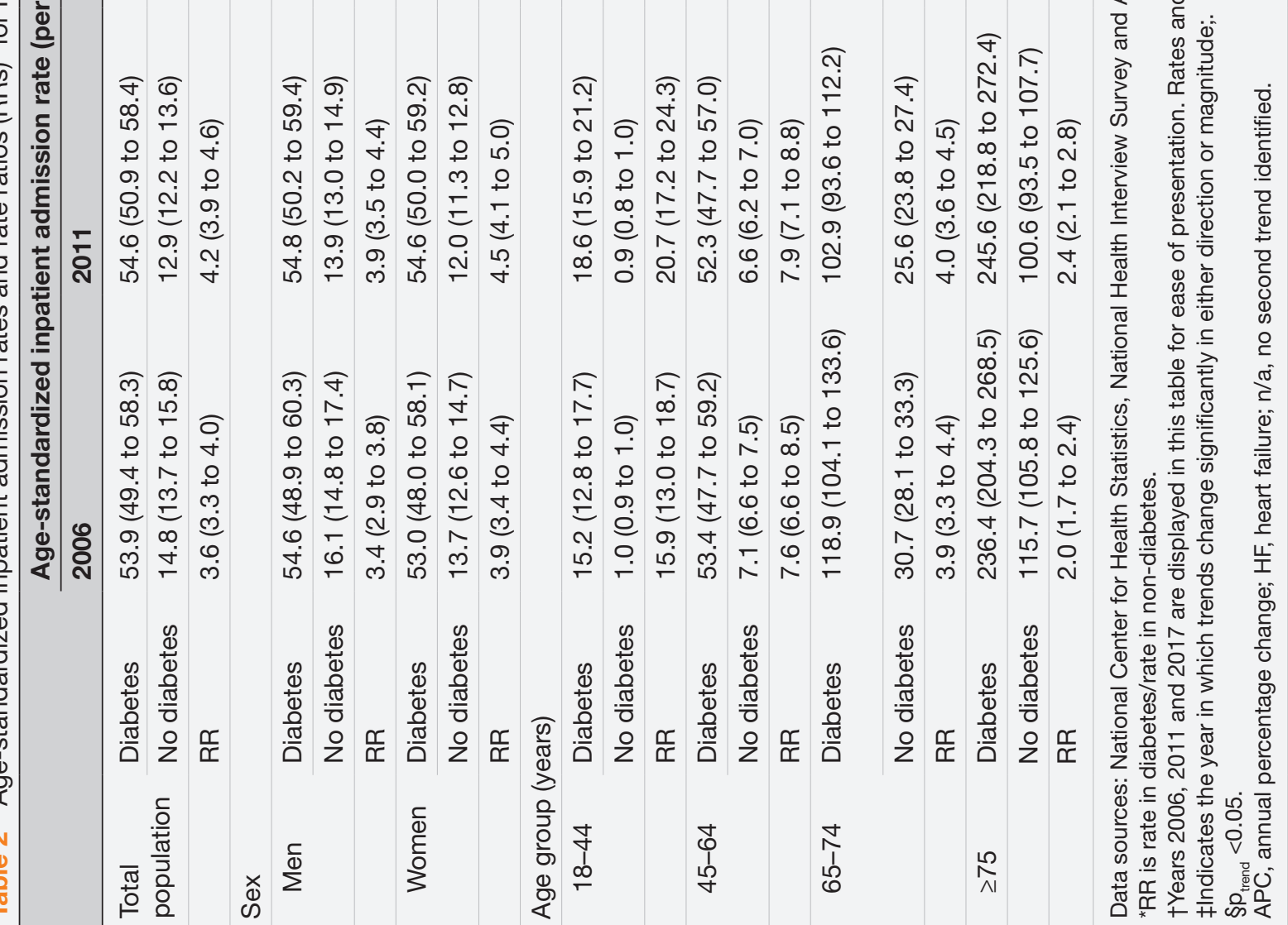




\section{A. NIS}

\section{i. Diabetes}

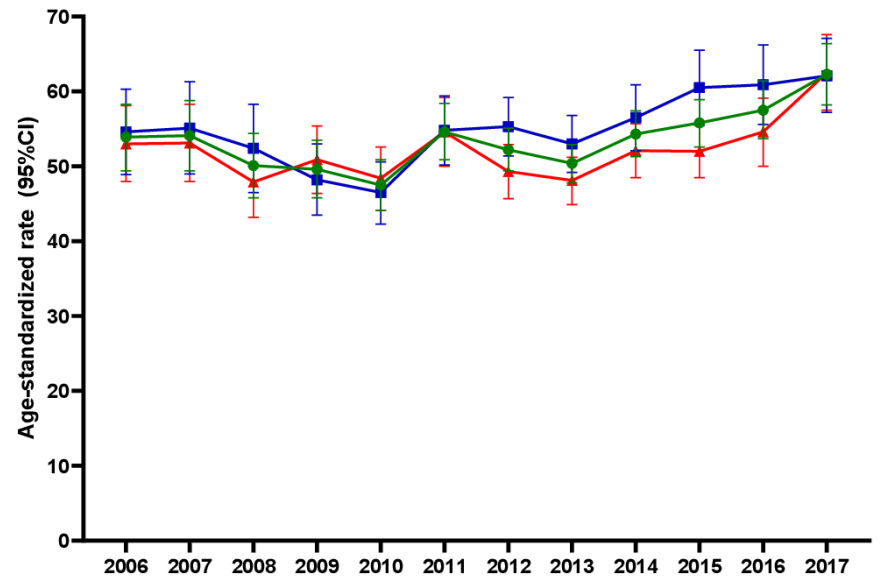

\section{B. NEDS}

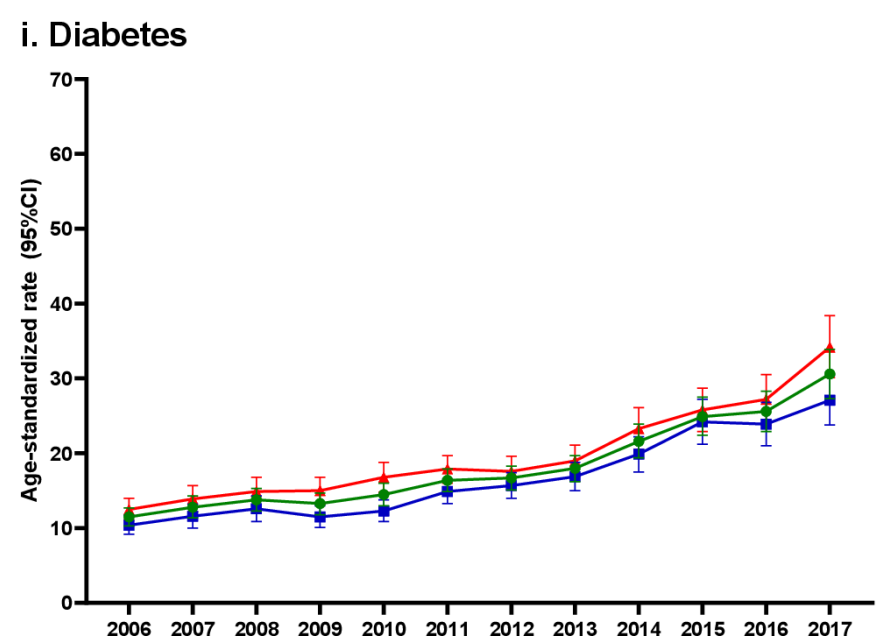

\section{ii. No Diabetes}

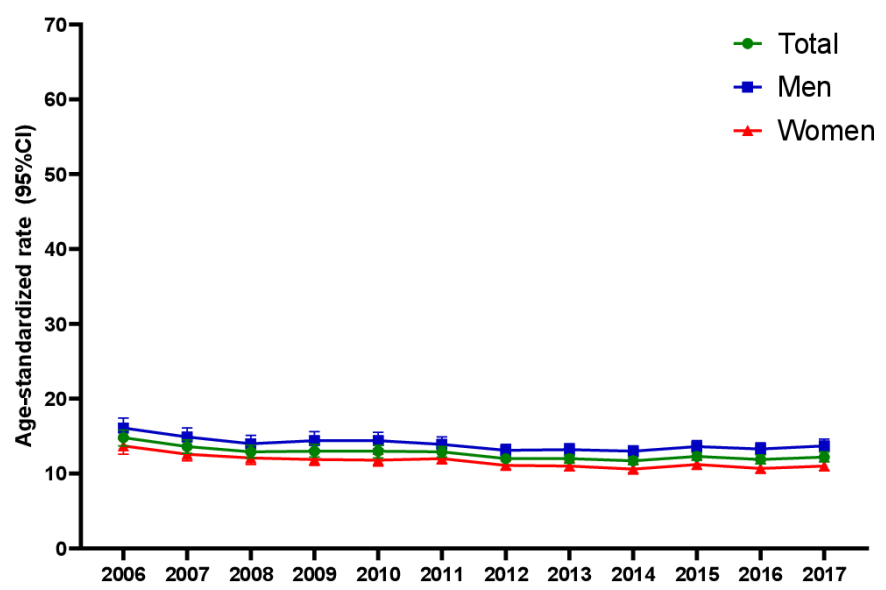

\section{ii. No Diabetes}

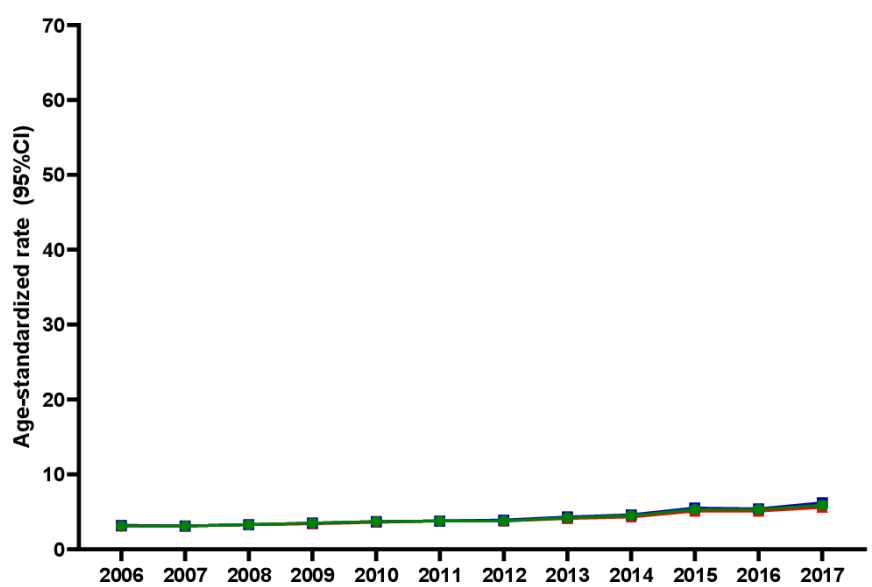

Figure 1 Age-standardized inpatient admission (NIS (A)) and ED visit (NEDS (B)) rates for HF in people with (i) versus without diabetes (ii) in the USA between 2006 and 2017. HF, heart failure; NEDS, Nationwide Emergency Department Sample; NIS, National Inpatient Sample.

This was driven by continued declines in HF rates among people without diabetes throughout the study period, while HF rates among people with diabetes plateaued from approximately 2010 onwards. For HF-related ED visits defined as the primary cause, the excess risk associated with diabetes also increased over time driven by increases in HF rates among people with, but not without, diabetes in the latter study period (online supplemental table 3).

\section{DISCUSSION}

In this study, we provide the first comprehensive summary of trends of HF-related inpatient admissions and nonadmitted ED visits in the USA among adults with and without diabetes and note several important findings. First, rates of HF-related inpatient admissions and ED visits were three to five times higher in adults with versus without diabetes, and this excess risk has increased over time. Second, while absolute rates remained lowest in the youngest age groups, the greatest relative increases in HF-related inpatient admissions and ED visits were observed in young adults with diabetes. Third, increases in HF-related utilization among adults with diabetes was observed in both inpatient and ED settings, suggesting broader underlying causes rather than a shift in treatment setting.

Our results are consistent with the few studies that have reported changes in HF incidence over time. In the USA, a NIS-based study reported a $3.6 \%$ annual decline in $\mathrm{HF}$ inpatient admissions among adults $\geq 35$ years with diabetes between 1998 and $2014 .^{13}$ This decline was likely driven by significant decreases in the earlier period (ie, 1998-2006) and explains why we, in contrast, observed a non-significant decline in HF-related inpatient admissions from 2006 to 2013. Another study, also using the NIS, reported an overall $38.9 \%$ decline in primary HF admissions in people with diabetes between 1995 and $2015 .^{12}$ This decline also appeared to be driven by reductions in 


\section{A. NIS}
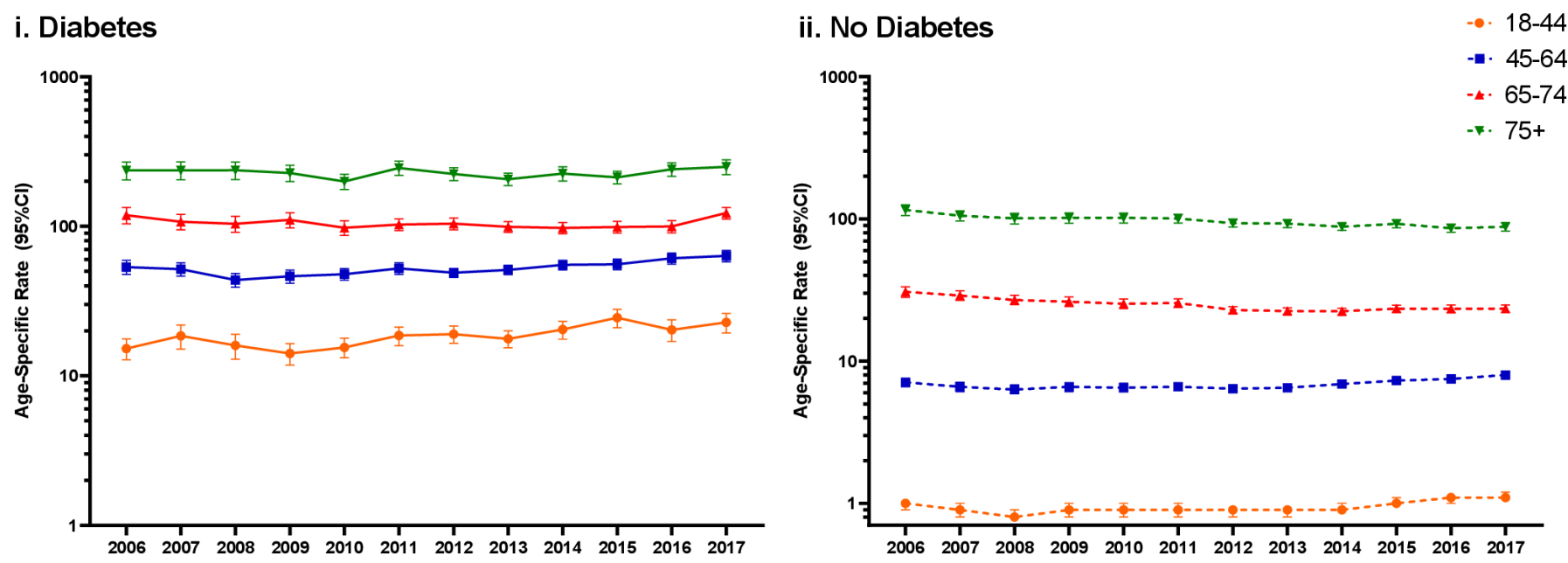

\section{B. NEDS}
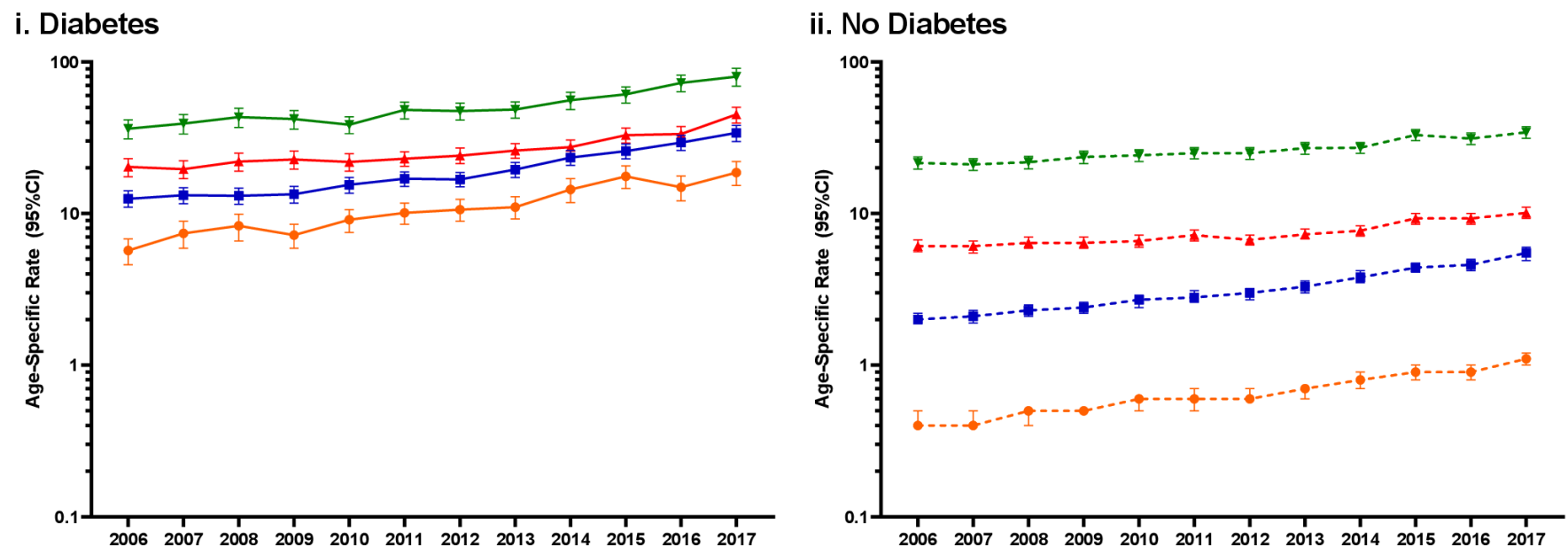

Figure 2 Age-specific inpatient admission (NIS (A)) and ED visit (NEDS (B)) rates for HF in people with (i) versus without diabetes (ii) in the USA between 2006 and 2017. HF, heart failure; NEDS, Nationwide Emergency Department Sample; NIS, National Inpatient Sample.

the earlier study period as non-significant increases were observed between 2013 and 2015. ${ }^{12}$ In Spain, a significant $5.4 \%$ annual increase in HF hospitalizations was observed between 1997 and 2010 in patients with diabetes, broadly similar to findings in the current study. ${ }^{23}$ However, the NIS-based studies and the Spanish study did not compare changes in HF incidence in people with versus without diabetes. This comparison is necessary to understand whether diabetes is an underlying cause of changing $\mathrm{HF}$ rates and to develop targeted interventions to reduce the HF burden in this subpopulation. Only one other study has compared rates of HF hospitalizations in people with versus without diabetes. In Sweden, a 29\% decrease in $\mathrm{HF}$ hospitalization rates, defined as primary of contributory cause, among persons with type 2 diabetes was observed between 1999 and 2013, and this decline was greater than what was observed for people without type 2 diabetes. ${ }^{24}$ Unfortunately, data beyond 2013 were not available, and thus, it remains to be elucidated whether the recent increase in HF hospitalizations seen in our US data is also occurring in other populations and settings.

The increasing rates of HF among people with diabetes, especially young adults with diabetes, are consistent with a recent resurgence of other diabetes-related complications in the USA. ${ }^{25}$ Between 2010 and 2015, national data show increases in lower extremity amputations (LEAs) ${ }^{26}$ and hyperglycaemic crises among adults with diabetes, ${ }^{27}$ while long-term declines in end-stage renal disease, acute myocardial infarction (AMI) and stroke have stalled. ${ }^{25}$ These trends appear to be driven by increases in young (aged 18-44 years) and middle-aged (aged 45-64 years) adults, among whom the risk of hyperglycaemic crisis, AMI, stroke and LEAs each increased by more than $25 \%$ between 2010 and $2015 .^{25}$ We add to this growing body of literature that increases in HF also disproportionally affect young people with diabetes at or around the same time. There are several possible reasons to explain this observed increase. First, we have observed a changing 


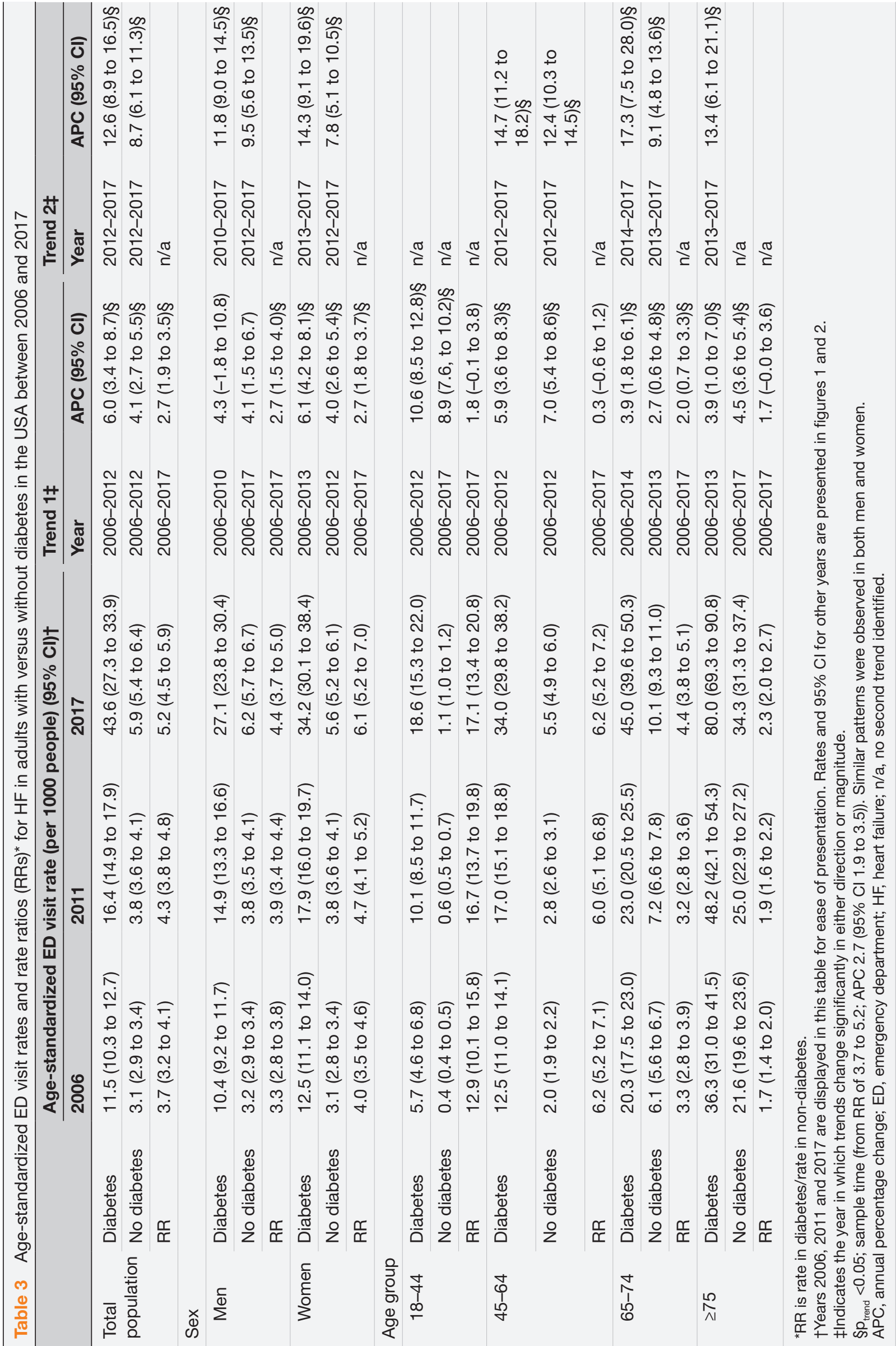


profile of newly identified diabetes cases that are more obese and may have more poorly managed risk factors (eg, blood pressure and lipids) as compared with earlier years, particularly among younger adults. ${ }^{4}$ Second, a longer average duration of diabetes may be leading to a shift in risk of complications. Third, the younger age group may include a larger relative proportion of type 1 diabetes who may be at increased risk for HF. However, accumulating evidence suggests that diabetes complication rates may be higher in young adults with type 2 diabetes as compared with type 1 diabetes. ${ }^{28}$ Fourth, changes in healthcare policy such as the introduction of high-deductible health plans have led to reductions in early preventive care in people with diabetes. ${ }^{29}{ }^{30}$ Fourth, increased costs of insulin and other diabetes medications may have led patients to cut back on treatment to minimize costs, thus exposing them to increased risk for complications including $\mathrm{HF}^{31}$ Last, in 2012, the US Centers for Medicare and Medicaid Services implemented the Hospital Readmissions Reduction Program, which financially penalized hospitals with high 30-day readmission rates for $\mathrm{HF}^{32}$ The role of this policy in influencing HF trends in the current study is unclear as NIS and NEDS do not identify hospital readmission. Overall, it is most likely that a combination of these factors explains the increases in HF-related ED visits and hospitalization among US adults with diabetes.

The results of this study offer important implications for public health and healthcare practice. First, in this study, we show that diabetes is associated with an almost fivefold increased risk for HF-related inpatient and non-admission ED visits. The continued increase in the prevalence of diabetes is likely to increase the number of people with HF in the future and will have important implications for both outpatient and hospital burdens, pharmacotherapies and resource allocation. Second, we hypothesize that increasing risk for HF may lead to an increase in subsequent HF-related mortality with some early evidence to support this hypothesis. For example, Cheng $e t a \hat{l}^{33}$ reported an increase in HF-related mortality among young US adults with diabetes between 1988 and 2015, despite mortality rates for several other CVDs declining in that time, and an Australian study reported no change in HF-related mortality despite declines for other CVD outcomes. ${ }^{34}$ Third, improved awareness by healthcare providers that diabetes is an important risk factor for $\mathrm{HF}$ might stimulate more intensive and focused prevention and management opportunities. For example, post hoc analysis of the Steno-2 trial in Denmark demonstrated a reduction in HF hospitalizations among patients with diabetes receiving intensive (vs conventional) therapy. ${ }^{35}$ Furthermore, emerging trial data of sodium-glucose cotransporters 2 (SGLT2) inhibitors show promising findings for HF. For example, randomized trials of SGLT2 inhibitors (vs placebo) have shown a pooled $31 \%$ reduction in HF hospitalizations in type 2 diabetes patients at high risk of CVD, ${ }^{36}$ as well as improved outcomes among those with existing diabetes and HF. ${ }^{37}$ Real-world studies, such as CVD-REAL (Comparative Effectiveness of Cardiovascular Outcomes in New Users of SGLT-2 Inhibitors), have also demonstrated the positive effects of SGLT-2 inhibitors in HF prevention in patients with type 2 diabetes, irrespective of atherosclerotic disease status. ${ }^{38} 39$

This is the largest study to explore rates of HF over time in USA adults with and without diabetes in two nationally representative patient datasets. Nonetheless, there are limitations to be considered. First, NIS and NEDS represent hospital discharges, not individual persons and therefore may include multiple hospital stays for some persons. This may lead to an increase in population-based rates, especially in certain subpopulations at higher risk for recurrence, including those with diabetes. ${ }^{40}$ However, the primary objective of this study was to examine changes in HF admissions over time in people with versus without diabetes. To that end, and in the absence of contrary data, we assume that the risk of readmission in people with versus without diabetes remained constant during the study period and readmissions are, therefore, unlikely to impact our key conclusions. Second, because of the inability to differentiate diabetes type in the NHIS survey data, we were not able to report trends in HF by diabetes type. Therefore, all types of diabetes are included in the current analysis with the assumption that the vast majority $(\sim 90 \%-95 \%)$ have type 2 diabetes. ${ }^{41}$ In addition, the NHIS is self-reported and does not include undiagnosed diabetes and thus likely underestimates the number of people with diabetes in the population. Furthermore, the underlying characteristics of people with diagnosed diabetes could be changing over time. However, there have not been adequate data or studies to characterize such changes. Third, a shift from ICD-9-CM to ICD-10-CM in October 2015 may have affected our observed rates. However, observed changes in trends occurred before this period, and therefore, it is unlikely that this coding shift influenced the overall patterns that we observed in this study. Furthermore, coding changes do not explain differential increases in people with versus without diabetes and in younger versus older adults. Fourth, admissions for hypertensive heart disease with $\mathrm{HF}$ were not included in the current analysis. Fifth, NIS and NEDS do not report HF stages and we were unable to explore differential impacts of diabetes on HF stages, though this is an important future direction. Sixth, location (urban/ rural) and poverty status, although available in NHIS, were not categorized in the same way in NEDS and NIS, so these factors were excluded from rate calculations. In addition, the race/ethnicity variable in NIS was incomplete prior to 2012, and so trends were not calculated by race/ethnicity. Finally, this is a descriptive observational study designed to assess the relative burden of $\mathrm{HF}$ hospitalizations in people with versus without diabetes over time. Future studies with more appropriate datasets (ie, with individual level data) are needed to tease out the underlying mechanisms with which diabetes leads 
to an increase in HF hospitalization, particularly among young adults.

\section{CONCLUSIONS}

In this nationally representative study, we show that: (1) rates of HF-related inpatient admissions increased in adults with, but not without, diabetes and (2) rates of HF-related ED visits increased in adults with and without diabetes, but absolute and relative increases were greater in adults with diabetes; and (3) the greatest relative increases in HF-related inpatient admissions and nonadmission ED visits was seen among young adults with diabetes. More detailed and subnational data analyses may help to investigate the aetiology and determine clinical and public health strategies to address these growing burdens.

\section{Author affiliations}

${ }^{1}$ Department of Surgery, Emory University School of Medicine, Atlanta, Georgia, USA ${ }^{2}$ Department of Medicine, Emory University School of Medicine, Atlanta, Georgia, USA

${ }^{3}$ Division of Diabetes Translation, Centers for Disease Control and Prevention, Atlanta, Georgia, USA

${ }^{4}$ Division of Cardiology, Emory University School of Medicine, Atlanta, Georgia, USA ${ }^{5}$ Global Diabetes Research Center, Rollins School of Public Health Emory University, Atlanta, Georgia, USA

${ }^{6}$ Department of Family and Preventive Medicine, Emory University School of Medicine, Atlanta, Georgia, USA

Acknowledgements The authors would like to thank the women and men who participated in the National Health Interview Survey (NHIS), as well as all the staff involved at the NCHS and HCUP for study design, data collection and data dissemination.

Contributors JLH designed the study, conducted the analyses, interpreted the results and wrote the manuscript. SRB and IH conducted the analysis, contributed to interpretation and reviewed the manuscript. LS provided clinical input, contributed to interpretation and reviewed the manuscript. RJ, REP and MKA contributed to interpretation and reviewed the manuscript. KMVN conceptualized the manuscript, contributed to interpretation and reviewed the manuscript. JLH is the guarantor of this work and takes responsibility for the integrity of the data and final responsibility for the decision to submit for publication.

Funding Research reported in this publication was supported by the National Institute of Diabetes and Digestive and Kidney Diseases of the National Institutes of Health under Award Number P30DK111024 and the National Institute on Minority Health and Health Disparities grant U01MD010611. The findings and conclusions in this report are those of the authors and do not necessarily represent the official position of the Centers for Disease Control and Prevention or the National Institutes of Health.

\section{Competing interests None declared.}

Patient consent for publication Not applicable.

Ethics approval The NHIS, NIS and NEDS databases are publicly available and do not contain direct personal identifiers and are therefore exempt from review by the institutional review boards of the Centers for Disease Control and Prevention and Emory University.

Provenance and peer review Not commissioned; externally peer reviewed.

Data availability statement Data are available in a public, open access repository. The data that support the findings of this study are publicly available from the National Center for Health Statistics and the Agency for Healthcare Research and Quality. All data are deidentified.

Supplemental material This content has been supplied by the author(s). It has not been vetted by BMJ Publishing Group Limited (BMJ) and may not have been peer-reviewed. Any opinions or recommendations discussed are solely those of the author(s) and are not endorsed by BMJ. BMJ disclaims all liability and responsibility arising from any reliance placed on the content. Where the content includes any translated material, BMJ does not warrant the accuracy and reliability of the translations (including but not limited to local regulations, clinical guidelines, terminology, drug names and drug dosages), and is not responsible for any error and/or omissions arising from translation and adaptation or otherwise.

Open access This is an open access article distributed in accordance with the Creative Commons Attribution Non Commercial (CC BY-NC 4.0) license, which permits others to distribute, remix, adapt, build upon this work non-commercially, and license their derivative works on different terms, provided the original work is properly cited, appropriate credit is given, any changes made indicated, and the use is non-commercial. See: http://creativecommons.org/licenses/by-nc/4.0/.

\section{ORCID IDs}

Jessica L Harding http://orcid.org/0000-0002-6664-8630

Stephen R Benoit http://orcid.org/0000-0003-3455-133X

Lakshmi Sridharan http://orcid.org/0000-0002-4855-4892

K M Venkat Narayan http://orcid.org/0000-0001-8621-5405

\section{REFERENCES}

1 Authors/Task Force Members, Rydén L, Grant PJ, et al. ESC guidelines on diabetes, pre-diabetes, and cardiovascular diseases developed in collaboration with the EASD: the task force on diabetes, pre-diabetes, and cardiovascular diseases of the European Society of cardiology (ESC) and developed in collaboration with the European association for the study of diabetes (EASD). Eur Heart $J$ 2013;34:3035-87.

2 International Diabetes Federation. IDF diabetes atlas. 9th edn. Brussels, Belgium: International Diabetes Federation, 2019. https:// www.diabetesatlas.org

3 Centers for Disease Control and Prevention. National diabetes statistics report. Atlanta, GA: Centers for Disease Control and Prevention, U.S. Dept of Health and Human Services, 2020. https:// www.cdc.gov/diabetes/data/statistics-report/index.html

4 Ali MK, Bullard KM, Saaddine JB, et al. Achievement of goals in U.S. diabetes care, 1999-2010. N Engl J Med 2013;368:1613-24.

5 Ali MK, Bullard KM, Gregg EW, et al. A cascade of care for diabetes in the United States: visualizing the gaps. Ann Intern Med 2014:161:681-9.

6 Booth GL, Kapral MK, Fung K, et al. Recent trends in cardiovascular complications among men and women with and without diabetes. Diabetes Care 2006;29:32-7.

7 Jung $\mathrm{CH}$, Chung JO, Han K, et al. Improved trends in cardiovascular complications among subjects with type 2 diabetes in Korea: a nationwide study (2006-2013). Cardiovasc Diabetol 2017;16:1.

8 Levi F, Lucchini F, Negri E, et al. Trends in mortality from cardiovascular and cerebrovascular diseases in Europe and other areas of the world. Heart 2002;88:119-24.

9 Shah AD, Langenberg C, Rapsomaniki E, et al. Type 2 diabetes and incidence of cardiovascular diseases: a cohort study in 1.9 million people. Lancet Diabetes Endocrinol 2015;3:105-13.

10 Taylor KS, Heneghan CJ, Farmer AJ, et al. All-Cause and cardiovascular mortality in middle-aged people with type 2 diabetes compared with people without diabetes in a large U.K. primary care database. Diabetes Care 2013;36:2366-71.

11 Vamos EP, Millett C, Parsons C, et al. Nationwide study on trends in hospital admissions for major cardiovascular events and procedures among people with and without diabetes in England, 2004-2009. Diabetes Care 2012;35:265-72.

12 Honigberg MC, Patel RB, Pandey A, et al. Trends in hospitalizations for heart failure and ischemic heart disease among US adults with diabetes. JAMA Cardiol 2021;6:354-7.

13 Burrows NR, Li Y, Gregg EW, et al. Declining rates of hospitalization for selected cardiovascular disease conditions among adults aged $\geq 35$ years with diagnosed diabetes, U.S., 1998-2014. Diabetes Care 2018;41:293-302.

14 Gregg EW, Cheng YJ, Srinivasan M, et al. Trends in cause-specific mortality among adults with and without diagnosed diabetes in the USA: an epidemiological analysis of linked national survey and vital statistics data. Lancet 2018;391:2430-40.

15 Agency for HealthCare Research and Quality. Nationwide HCUP databases, 2021. Available: https://www.hcup-us.ahrq.gov/ databases.jsp

16 Clinical Classifications Software (CCS) for ICD-10-PCS (beta version). Healthcare Cost and Utilization Project (HCUP), 2019Agency for Healthcare Research and Quality, Rockville, MD. Available: www.hcup-us.ahrq.gov/toolssoftware/ccs10/ccs10.jsp [Accessed 7 October 2021].

17 Elixhauser A, Heslin KC, Owens PL. Healthcare cost and utilization project (HCUP) recommendations for reporting trends using ICD- 
9-CM and ICD-10-CM/PCS data. Agency for Healthcare Research and Quality, 2017. Available: https://www.hcup-us.ahrq.gov/ datainnovations/icd10_resources.jsp

18 National Center for Health Statistics. Survey description, National health interview survey Hyattsville, Maryland, 2017. Available: ftp:// ftp.cdc.gov/pub/Health_Statistics/NCHS/Dataset_Documentation/ NHIS/2017/srvydesc.pdf

19 Centers for Disease Control and Prevention. National diabetes statistics report. Atlanta, GA: Centers for Disease Control and Prevention, U.S. Dept of Health and Human Services, 2020.

20 Localio AR, Margolis DJ, Berlin JA. Relative risks and confidence intervals were easily computed indirectly from multivariable logistic regression. J Clin Epidemiol 2007;60:874-82.

21 Houchens RL RD, Elixhauser A. Using the HCUP national inpatient sample to estimate trends. HCUP methods series report \# 200605 online. U.S. agency for healthcare research and quality, 2018. Available: http://www.hcup-us.ahrq.gov/reports/methods/methods. jsp

22 National Cancer Institute. Joinpoint trend analysis software, 2018. Available: https://surveillance.cancer.gov/joinpoint/

23 Lara-Rojas CM, Pérez-Belmonte LM, López-Carmona MD, et al. National trends in diabetes mellitus hospitalization in Spain 19972010: analysis of over 5.4 millions of admissions. Eur J Intern Med 2019;60:83-9.

24 Rawshani A, Rawshani A, Franzén S, et al. Mortality and cardiovascular disease in type 1 and type 2 diabetes. N Engl J Med 2017;376:1407-18.

25 Gregg EW, Hora I, Benoit SR. Resurgence in diabetes-related complications. JAMA 2019;321:1867-8.

26 Geiss LS, Li Y, Hora I, et al. Resurgence of diabetes-related nontraumatic lower-extremity amputation in the young and middleaged adult U.S. population. Diabetes Care 2019;42:50-4.

27 Benoit SR, Hora I, Pasquel FJ, et al. Trends in emergency department visits and inpatient admissions for hyperglycemic crises in adults with diabetes in the U.S., 2006-2015. Diabetes Care 2020;43:1057-64.

28 Magliano DJ, Sacre JW, Harding JL, et al. Young-onset type 2 diabetes mellitus - implications for morbidity and mortality. Nat Rev Endocrinol 2020;16:321-31.

29 Carls G, Huynh J, Tuttle E, et al. Achievement of glycated hemoglobin goals in the US remains unchanged through 2014 Diabetes Ther 2017;8:863-73.
30 Wharam JF, Zhang F, Eggleston EM, et al. Effect of high-deductible insurance on High-Acuity outcomes in diabetes: a natural experiment for translation in diabetes (NEXT-D) study. Diabetes Care 2018;41:940-8.

31 Riddle MC, Herman WH. The cost of diabetes Care-An elephant in the room. Diabetes Care 2018;41:929-32.

32 Wadhera RK, Joynt Maddox KE, Wasfy JH, et al. Association of the hospital readmissions reduction program with mortality among Medicare beneficiaries hospitalized for heart failure, acute myocardial infarction, and pneumonia. JAMA 2018;320:2542-52.

33 Cheng YJ, Imperatore G, Geiss LS, et al. Trends and disparities in cardiovascular mortality among U.S. adults with and without selfreported diabetes, 1988-2015. Diabetes Care 2018;41:2306-15.

34 Sacre JW, Harding JL, Shaw JE, et al. Declining mortality in older people with type 2 diabetes masks rising excess risks at younger ages: a population-based study of all-cause and cause-specific mortality over 13 years. Int J Epidemiol 2021;50:1362-72.

35 Oellgaard J, Gæde P, Rossing P, et al. Reduced risk of heart failure with intensified multifactorial intervention in individuals with type 2 diabetes and microalbuminuria: 21 years of follow-up in the randomised Steno-2 study. Diabetologia 2018;61:1724-33.

36 Kumar K, Kheiri B, Simpson TF, et al. Sodium-Glucose cotransporter-2 inhibitors in heart failure: a meta-analysis of randomized clinical trials. Am J Med 2020;133:e625-30.

37 McMurray JJV, Solomon SD, Inzucchi SE, et al. Dapagliflozin in patients with heart failure and reduced ejection fraction. $N$ Engl $J$ Med 2019;381:1995-2008.

38 Kosiborod M, Cavender MA, Fu AZ, et al. Lower risk of heart failure and death in patients initiated on sodium-glucose cotransporter-2 inhibitors versus other glucose-lowering drugs: the CVD-REAL study (comparative effectiveness of cardiovascular outcomes in new users of sodium-glucose cotransporter-2 inhibitors). Circulation 2017;136:249-59.

39 Cavender MA, Norhammar A, Birkeland KI, et al. SGLT-2 inhibitors and cardiovascular risk: an analysis of CVD-REAL. J Am Coll Cardiol 2018;71:2497-506.

40 Patil S, Shah M, Patel B, et al. Readmissions among patients admitted with acute decompensated heart failure based on income Quartiles. Mayo Clin Proc 2019;94:1939-50.

41 Centers for Disease Control and Prevention. National diabetes statistics report. Atlanta, GA: Centers for Disease Control and Prevention, 2017. 